\title{
The statistical presentation of seismicity for appreciating seismic hazard
}

\author{
E Jones Beck Engineering Pty Ltd, Australia \\ D Beck Beck Engineering Pty Ltd, Australia \\ E Villaescusa Western Australian School of Mines, Curtin University/CRC Mining, Australia
}

\begin{abstract}
In seismically active mines, the engineer responsible must certify that the additional hazard from dynamic deformation will not cause the total work place risk of injury to be greater than the accepted norm, or is not in itself excessive. This duty requires the engineer to make sufficient, quantitative forecasts of the likelihood, and eventually consequence, of hazardous dynamic deformation and seismic events.

Various methods are in use to calculate, or quantify, the dynamic and or seismic hazard in work places, but none encompass all of the classes of hazard. Few methods caution the user to consider the range of potential mechanisms of seismic hazard in a mine. A range of approaches and intuitive understanding of their limitations is needed if seismically active mines are to be made safer.

This paper discusses a statistical method for the determination and representation of seismic hazard in mines to assist the engineer to appreciate dynamic rock related hazard levels. The intent is to highlight some methods of data presentation that make it easier for non-experts to appreciate evolving seismic risks in operating mines and to learn as much as possible about the stability of a mine by interrogating seismic data.
\end{abstract}

\section{Introduction}

As mines deepen, deformation and rock mass damage around excavations will increase. In most areas of the mine equilibrium following excavation will be reached by a quasi-static process. However, some damage in the skin of mine excavations occurs rapidly. Where accelerations in an excavation are substantive, momentum and acceleration must be considered in a stability analysis.

The degradation in the stiffness of the rock mass of the mine is also associated with a release of elastic energy. If the value of the released energy is higher than the energy dissipated by irreversible damage to the rock mass, the surplus is emitted into the surrounding rock mass. These release events are the seismic events measured by the mine's seismic system.

Most seismic events cause no observable damage. However, the existence of seismic events indicates the occurrence of damage to the rock mass, and this in turn generates a hazard to the underground workplace. When considering seismic hazard, it is the rapid, uncontrolled, and sometimes violent, release of material that is of most concern in underground workplaces. This form of seismic damage, referred to as dynamic damage, can occur as a result of two mechanisms:

- Dynamic damage caused by evolving stress states, arising as the system comes to equilibrium after a change. The evolving stress states initially cause isolated areas of instability observed as regions of nucleating seismicity. These regions continue to grow, and eventually coalesce generating a system instability capable of causing dynamic damage.

- Dynamic damage resulting from transient stress waves intersecting an existing excavation. This type of dynamic damage is caused by larger events in close proximity to workings, generating sufficient conditions for additional dynamic instability, either by causing additional yield and dilation or by shaking down loose material. 
In both mechanisms there is a correlation with seismicity. The engineer needs to appreciate the levels of seismicity in each work area and interpret the consequences for the mining plan. Additionally, the engineer must be able to convert observations made using a seismic system to a statistical or probabilistic assessment of the likelihood that a worker in a given location will be exposed to a hazard within a time frame. In other words, how frequently will a worker be within a distance $x$, of an event of magnitude $y$, within time frame $t$ ?

\section{Hazard domains}

There is a particular problem with mines accumulating a consistent data set representative of the current range of conditions throughout a mine. It is a difficult task, as our knowledge of the environment is poor prior to the start of mining, and the data required to understand orebody continuity is at a lower resolution than that required to understand structural complexity affecting seismic risks.

A solution to the continuity problem is to divide the mine into hazard domains. Hazard domains are based upon meaningfully similar geology (strength and structure) conditions. The greater the number of equal basis observations in a given hazard domain, the higher the confidence in future predictions for that domain.

Additionally, the observations in each hazard domain need to be compared based upon similar geometry (void and time) conditions, otherwise forecasts will result in inaccurate representations and statistical outcomes. Observations of conditions in a particular hazard domain are also often limited to a period of weeks or months, depending on the speed of mining or proximity to mining activities.

The combination of the geology and geometry parameters result in small observation data sets in which statistically significant outcomes can be deduced. The effect of these limitations is lowered confidence, a risk of statistical bias, regression from the mean and poor resolution. These limitations can be worked with, however they need to be properly understood and conveyed to decision makers who rely on the forecasts.

The volume and discretisation of the sample space of each hazard domain will determine if the forecasts is useful:

- If the resolution is too low, it will not be useful to decision-makers.

- Each volume must describe a unique range of conditions of stress, strain, strength and structure.

- The size of the hazard domain has an influence on the interpretation of statistical results. Two examples are considered:

- Using a smaller sample space covering only a sub-element of the system. Instability may occur on the scale associated with large, damaging seismic events arises from interactions between different parts of a mine across a huge volume. At any particular time, only a part of that system may be actively yielding and may not be captured in the sample space.

- Using a large sample space covering multiple seismic hazards. Many systems for assessing seismic hazard use hazard domains that are too large. Large assessment volumes are used as an attempt to capture a large sample population. The result is that hazard levels are smeared and meaningful areas of excessive risk cannot be easily identified.

\section{$3 \quad$ Hazard management within hazard domains}

Hazard management within hazard domains can be defined by two phases, design and management. Mine design, ground support systems and hazard management protocols must be engineered for each hazard domain.

The first phase of hazard management must focus on design. That is, design of the mine layout and ground support systems so that the residual levels of risk are as low as possible. The mine should be engineered such that the ground support is not likely to be subject to excessive demand. 
In the second phase, the hazards within each domain need to be managed. This is undertaken using a system of measurements and observations to:

- Confirm that the assumptions of the design are correct.

- To disconfirm the presence of adverse factors.

- To confirm that the system is evolving as forecasted.

- To identify the evolving development of unsafe conditions.

The parameters being measured or observed must have an associated Trigger and Response Plan (TARP) developed for the particular environment. Ideally, a seismic hazard TARP should be tiered so as to not unduly impose restrictions on mining, while ensuring that there are procedures for dealing with the array of hazards. A properly tiered seismic hazard TARP will include caution, exclusion, and evacuation procedures.

A proposed configuration for a tiered seismic hazard TARP is shown in Figure 1. The three tiers correspond to cautionary, exclusion, and evacuation levels each triggered by different measured circumstances and each prescribing a different operational response:

- The caution level covers emergence of uncharacteristic seismicity or low levels of seismicity of an unexpected nature; but, which is not considered to be immediately hazardous. It would indicate areas that were otherwise thought to be at low-medium risk but requires that engineers investigate. A prolonged level of caution should be viewed as an area of potential instability and the mechanism for seismicity generation investigated.

- This exclusion tier is triggered when seismic conditions indicate a medium-high risk situation may be developing which could lead to dynamic hazards beyond an acceptable limit. Personnel are removed until the unsafe situation is disconfirmed. Engineers should understand the mechanism for generating the observed seismicity and a reporting structure in place for conveying the situation.

- The evacuation tier indicates that a hazardous event has occurred, or may be imminent. There is an exceptional risk involved and personnel exposure within these areas should not occur.

The triggers must be designed so that personnel are not exposed to unsafe events, for example, exclusion should have been implemented before the evacuation tier is triggered. Typical triggers could include:

- A single event greater than a given magnitude, set at a safe level but correlated with potential for larger events in the past.

- Multiple events greater than a given magnitude within a radius and time period of each other, again set at a safe level but a level which is either unexpected, or correlated with potential for larger events in the past.

- An event rate within a given radius and time period, again set at a safe level but a level which is either unexpected, or correlated with potential for larger events in the past.

Triggers must also be validated using real data, and frequently validated for each hazard domain. 


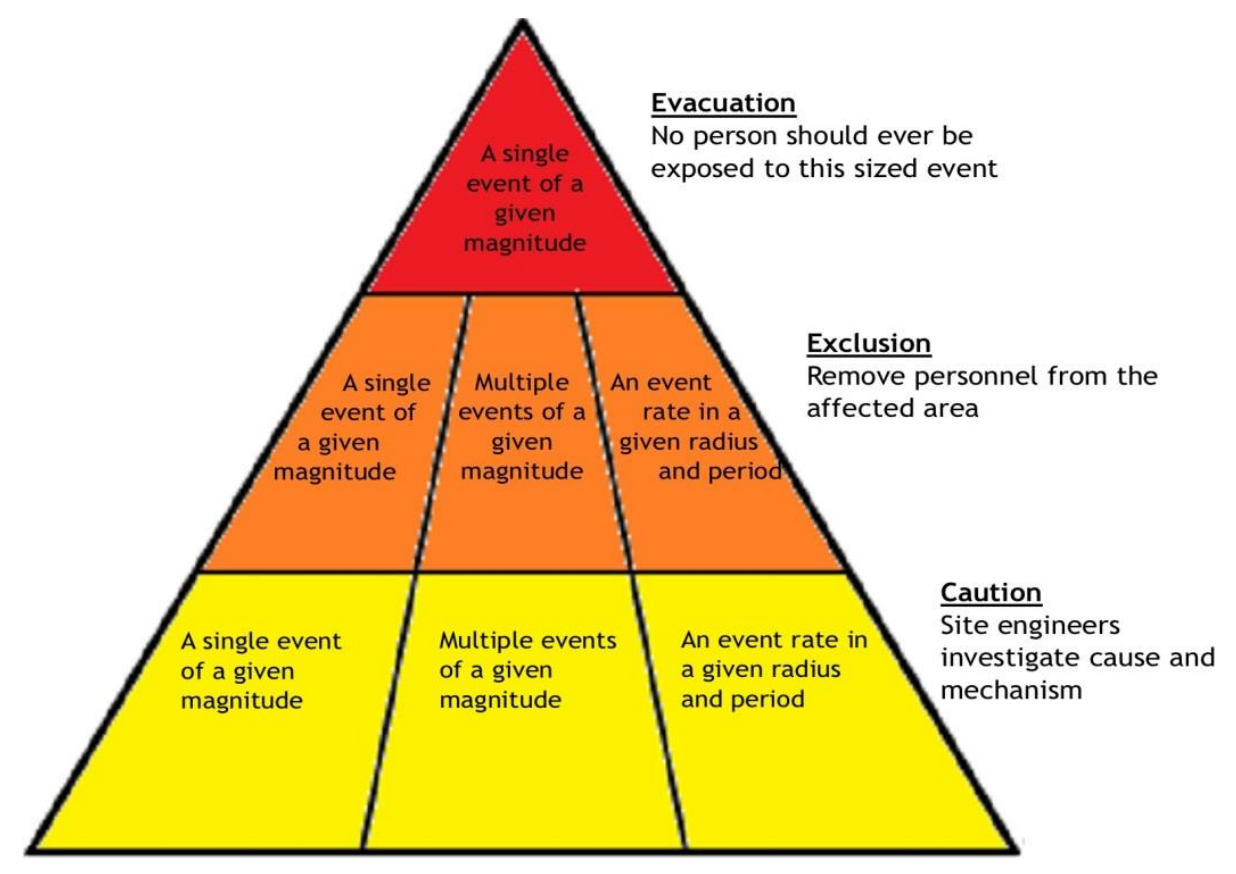

Figure 1 A proposed configuration for a tiered seismic hazard TARP

\section{$4 \quad$ Validating trigger criteria}

Through the use of gridding methods, event rates can be computed everywhere throughout the mine, and expressed as number of events per unit of time (quarters, months, etc.) within a given radius. This allows the data to be presented meaningfully using contours or isosurfaces showing different levels of seismic activity. Back-analysis of previous seismic episodes can be used to correlate event rates and inter-event distances to associated risk levels, and trigger criteria.

The search radius and event magnitudes can be iteratively tested and adjusted to achieve a best fit to the questions:

- What was the event rate throughout connected parts of the mine?

- What was the frequency of large but safe events within a certain distance and time before the largest events?

An additional trigger criteria is to set proximity limits:

- What proximity to a single large event, radius of multiple significant events, or event rate is permissible?

In answering this question there are some clearly defined distances to keep in mind, including:

- How far, and what type of damage occurred as a result of previous events?

- What distance does a discrete structure influence seismicity?

- What errors are there in significant/large event locations?

This technique effectively calibrates the trigger levels in the system, and can be used to validate the criteria. It also gives a visual presentation of regions of the mine where the criteria are being exceeded.

The quality of the data used for determining the trigger criteria should be as accurate as feasibly possible, and the limitations of the seismic array collecting the observations taken into consideration. As with all interpretations based upon seismic observations, it is important to ensure a quality dataset and the observations used within an analysis are within the known limitations of the seismic array. Caution should be used when excluding erroneous data; abnormal or outlying seismicity may be associated with an 
evolving instability and is required to be disproved as a potential mechanism, rather than assumed to be poorly observed data.

Whatever final solution is adopted, it must withstand comparison to past experience at the mine and similar mines elsewhere.

\section{$5 \quad$ Case study and implementation}

This method of presenting seismicity, seismic progression and indicating seismic hazard is still in its infancy and refinement is required on a site-to-site basis. It evolved as a method to analyse the seismic progression while conducting a back analysis for the mechanism behind a large seismic event at a deep and high stress mine in Australia. The daily implementation of the method was unable to be adopted by this mine due to operations ceasing. However, the method was further developed on a second deep and high stress mine in Australia, Mine A, where it is now in the process of being refined and implemented on a daily basis.

\subsection{Mine A case study - suggested TARP for daily implementation}

The refined version of the back-analysis was used for analysing the seismic progression, and formulation of the TARP, for Mine A in Australia. The TARP criteria and values suggested for implementation at Mine A are outlined in Figure 2.

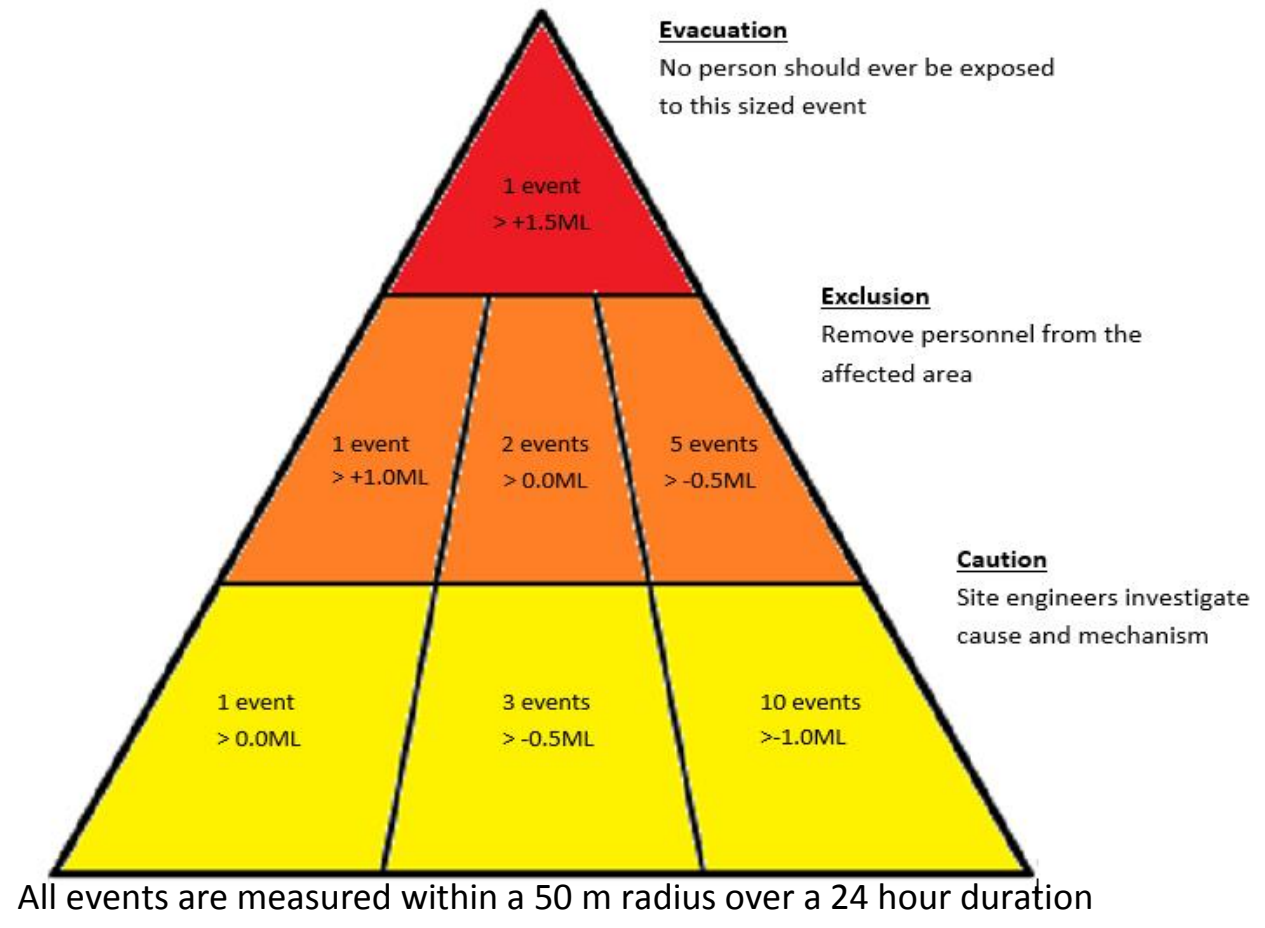

\section{Figure 2 Example of the TARP suggested for implementation at Mine A}

Following the above criteria, the back-analysis indicated that:

- A cautionary tier of risk would have been consistently triggered approximately 80 days prior to an event causing dynamic damage.

- Intermittent days of the exclusion tier were triggered for a period of 40 days prior to an eventual dynamic damage event.

- Consistent exclusion from the affected region occurred for a period of nine days prior to the eventual dynamic event. 
This method of seismic hazard analysis shows promise to be adopted on a daily basis. The event magnitudes, radius and rate are required to be reviewed regularly as mining conditions change.

\section{$6 \quad$ Further potential for implementation}

There exists the potential to further utilise this method beyond simple back-analysis and daily hazard forecasting and apply it to seismic potential of future mining based upon simulated energy release. The tiers, and associated triggers, presented in a TARP can reflect the conditions of a mine for any given calibrated time period. As such, simulated energy release can be correlated to periods of a known seismic response over a longer duration (i.e. quarters). The forward projections of simulated energy release can then be related to representative rates of seismicity for estimating the future mining conditions. Two examples are presented below for its use in distinguishing a block cave seismogenic zone and also the seismic response associated with a stoping operation.

The use in block caving is presented as an example in Figure 3 . In this case, simulated energy release (displayed as a volume rendered cloud) is correlated to the actual seismic response (displayed as a wireframe) for the corresponding period to define the seismogenic zone in a cave (Beck \& Putzar 2011). Such analysis can be undertaken for all events, or for events exceeding particular magnitude. Most importantly, it does not require the pre-imposition of zones, clusters or polygons of risk.

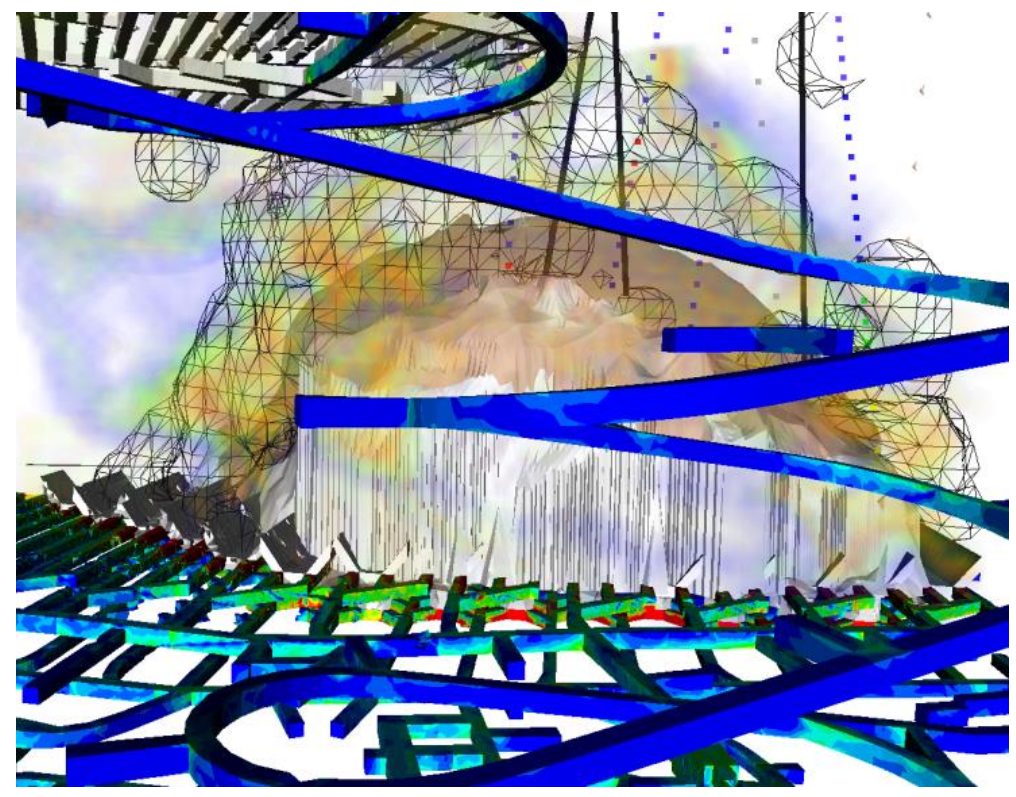

Figure 3 Measured seismogenic zone (wireframe) at an example mine. The wireframe is an isosurface of event occurrence, measured as number of events within a certain distance for a period

Figure 4 shows a similar principle, however, for a stoping operation (after Levkovitch et al. 2012). The measured seismic hazard level, corresponding to one of the tiers of the seismic hazard TARP, has been correlated with simulated rate of energy release. The matching of TARP tiers to simulated energy release allows for the interpretation, and quantification, of seismic potential to longer term quantitative forecasting. 


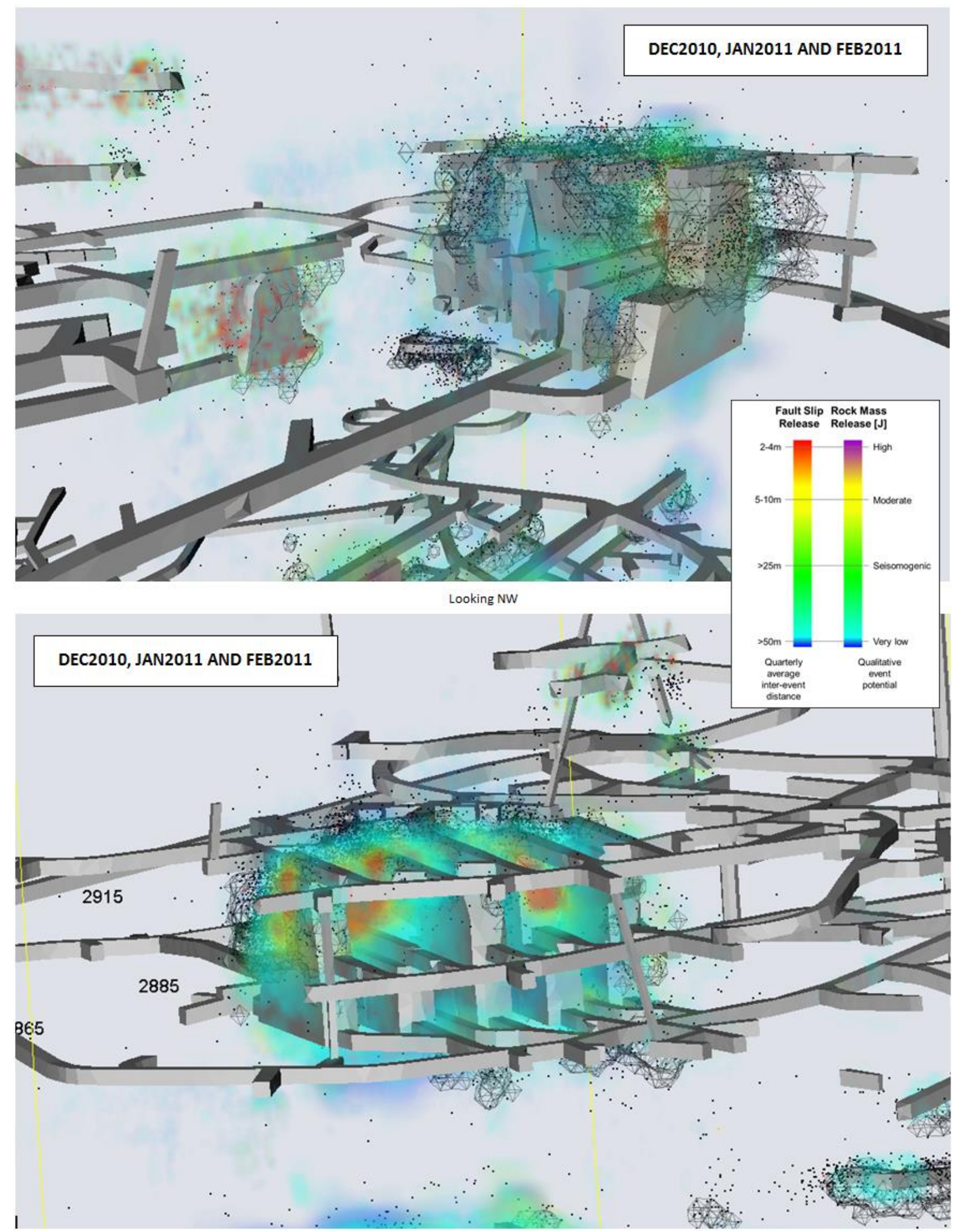

Looking S

Figure 4 Modelled rate of energy release versus seismicity for an example period from 


\section{Nickel Rim South Mine}

\section{$7 \quad$ Conclusions}

Most engineers use the term seismic hazard to encompass all dynamic rock related hazard.

A seismic hazard TARP, based on field data should include criteria and triggers that prevent personnel from being subject to unacceptable risk. These criteria must consider the resolution and precision of the data and the forecasting technique. The simpler these criteria; the easier they will be to comprehend and implement.

Ideally, these criteria should be able to be visualised by all decision makers and stake holders. Defining the criteria in terms of exposure to event of a certain size or a certain event rate permits simple visualisation and probabilistic definition of hazard and exposure.

For operational purposes a multi-tiered seismic hazard TARP comprising escalating levels of alarm and action is useful. The individual tiers can be set to reduce exposure to undue levels of risk. The method presented in this paper does not assume any predetermined hazard domains, and allows predetermined criteria to freely indicate areas satisfying tiers associated with increasing hazard and risk.

There exists the opportunity to further develop this method as a means of quantifying the seismic potential of associated simulated energy release across a number of mining methods. This in turn, can assist in the decision making and formulation of a safer mining plan based upon past and current mining conditions.

\section{References}

Beck, DA \& Putzar, G 2011, 'Coupled flow-deformation simulation for mine sale analysis of cave initiation and propagation', Proceedings of the 12th International Society of Rock Mechanics Conference, Taylor \& Francis Group, London.

Levkovitch, V, Beck, D \& Simser, B 2012, 'Explicit discontinuum simulation for probabilistic forecasting of fault slip and rock mass seismic potential', in Y Potvin (ed.), Proceedings of the Sixth International Seminar on Deep and High Stress Mining, Australian Centre for Geomechanics, Perth, pp. 373-399. 